\title{
Zamiaceae endémicas del Perú
}

\section{Blanca León ${ }^{1,2}$}

${ }^{1}$ Museo de Historia Natural, Av. Arenales 1256, Aptdo. 14-0434, Lima 14, Perú

2 Plant Resources Center, University of Texas at Austin, Austin TX 78712 EE.UU.

blanca.leon@mail.utexas.edu

\section{Resumen}

La familia Zamiaceae es reconocida en el Perú por presentar un género, Zamia, y cuatro especies (Brako \& Zarucchi, 1993; Stevenson, 2004), todas arbustivas, con tallos cortos menores de $2 \mathrm{~m}$ de alto o desde un tallo subterráneo. En este trabajo reconocemos dos endemismos, los cuales constituyen adiciones a la flora desde 1993 (Ulloa Ulloa et al., 2004). Estas especies endémicas han sido encontradas en bosques de las regiones de Bosques Muy Húmedos Premontanos y Húmedo Amazónico, entre los 100 y 700 m de altitud. Una de ellas se encuentra representada en una de las áreas naturales protegidas. Por lo atractivo de su follaje y valor ornamental, plantas en algunas poblaciones son extraídas ilegalmente para su comercialización.

Palabras claves: Zamiaceae, Zamia, cicadáceas, Perú, endemismo, especies endémicas.

\section{Abstract}

The Zamiaceae are represented in Peru by four species in the genus Zamia (Brako \& Zarucchi, 1993; Stevenson, 2004), all shrubs with stems less than $2 \mathrm{~m}$ high or with a subterranean stem. In this book we recognize two endemic species, both additions to the flora since 1993 (Ulloa Ulloa et al., 2004). These endemic species are found in lowland Amazonian forests and in the foothills of the montane forest, between 100 and $700 \mathrm{~m}$ elevation. One species has been registered within Peru's protected areas network. Due to their handsome foliage, species of this family are valued as ornamentals and some populations are affected by illegal extraction for commercial sale.

Keywords: Zamiaceae, Zamia, cycads, Peru, endemism, endemic species.

\section{Zamia macrochiera D.W. Stev.}

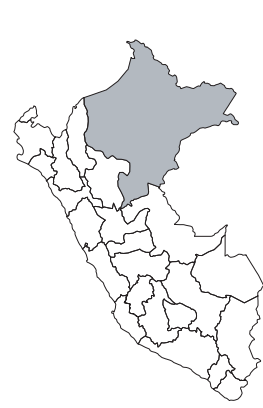

\section{EN, B1a}

Publicación: Cycad Classification: Concepts \& Recommendations: 185-186, f. 14.7. 2004.

Colección tipo: D.W. Stevenson et al. 1160 Herbarios: FTG, NY, U; AMAZ.

Nombre común: Desconocido.

Registro departamental: LO.

Regiones Ecológicas: BHA; $100-300$ m. SINANPE: Sin registro.

Herbarios peruanos: AMAZ (isotipo citado).

Observaciones: Hierba con tallo subterráneo, conocida de tres localidades, todas de la cuenca del Amazonas. La colección más reciente corresponde, al parecer, al ejemplar tipo, realizada en 1989.

\section{Zamia urep B. Walln.}

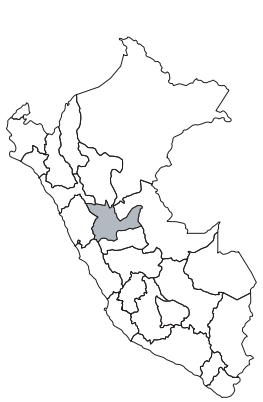

\section{CR, B1}

Publicación: Linzer Biol. Beitr. 28/2: 1056-1057, f.1. 1996.

Colección tipo: B. Wallnöfer 112-15788

Herbarios: LZ, W; USM!.

Nombre común: Desconocido.

Registro departamental: HU.

Regiones Ecológicas: BMHP, BHA; 260-700 m.

SINANPE: RCS

Herbarios peruanos: USM (isotipo+3).

Observaciones: Hierba conocida de colecciones provenientes de una localidad, las que fueron recolectados en laderas rocosas con poco suelo. Esta especie es dioica, las plantas femeninas son escasas en las poblaciones. La localidad tipo, en la Reserva Comunal El Sira, se ubica en una de las zonas más amenazadas por la expansión de agrícola y la deforestación. 